\title{
Estudantes de Medicina no enfrentamento da pandemia da Covid-19 no Brasil: reflexões éticas
}

\author{
Medical students in the COVID-19 pandemic response in Brazil: ethical reflections
}

\author{
Cleide Aparecida de Freitas ${ }^{1}$ \\ Gustavo Freitas Alves de Arruda ${ }^{2}$ \\ Giovanna Cecília Freitas Alves de Arruda' \\ Saulo Ferreira Feitosa \\ dracleidefreitas@gmail.com \\ gu14freitas@gmail.com \\ giovannacecilia11@gmail.com \\ sauloffeitosa@gmail.com
}

\section{RESUMO}

Introdução: Durante pandemias, as incertezas e a falta de evidências permitem que cada país conduza sua resposta da maneira que convencionar mais correta. Esse cenário abre oportunidade também para que medidas sejam aprovadas sem a devida análise ética, pela urgência implicada. Com isso, o objetivo deste estudo é promover uma abordagem hermenêutica das propostas do governo federal do Brasil para a inserção de estudantes de Medicina no combate à coronavirus disease 2019 (Covid-19) a partir de uma perspectiva ética.

Desenvolvimento: As resoluções governamentais, publicadas no Diário Oficial da União, foram debatidas à luz da Declaração Universal sobre Bioética e Direitos Humanos (DUBDH) e do Código de Ética do Estudante de Medicina (CEEM), porque aquela pauta a discussão em uma bioética plural, multi, inter e transdisciplinar e este traz orientações destinadas ao grupo populacional estudado. Para melhor estruturar a discussão, as principais medidas foram subdivididas em três seções: "Sobre a avaliação de risco"; "Sobre a participação dos alunos do quinto e sexto anos"; "Sobre a antecipação de colação de grau". Na primeira, propôs-se a elaboração de alternativas para sua participação de modo remoto ou sem contato direto com os pacientes, a fim de garantir a integridade dos estudantes e maximizar os efeitos positivos com o mínimo de prejuízos. Em seguida, avaliaram-se a prevista obrigatoriedade de adesão dos alunos dos últimos anos do curso de graduação e a possibilidade de substituição da carga horária do estágio curricular obrigatório pela participação na ação estratégica "O Brasil Conta Comigo". Por último, questionaram-se a antecipação de formatura e a garantia de que os recém-graduados possuam os conhecimentos e a perícia necessários à profissão médica.

Conclusões: Para o combate eficaz à doença, é necessária uma estruturação coletiva das ações adotadas, beneficiando-se das capacidades que os estudantes já oferecem, com respeito às suas limitações, vulnerabilidades e liberdades. Deve-se ressaltar que quaisquer decisões éticas no contexto da medicina e das futuras gerações de profissionais podem ter repercussões inquantificáveis para esses indivíduos, seus pacientes e suas comunidades, devendo-se ter a garantia de que os benefícios serão os melhores e maiores possíveis.

Palavras-chave: Educação Médica; Estudantes de Medicina; Bioética; Infecções por Coronavírus; Pandemias.

\begin{abstract}
Introduction: In times of pandemics, the uncertainties and lack of evidence allow each country to conduct its response as it deems the most appropriate. This setting also facilitates the approval of public measures without adequate ethical analysis, due to its inherent urgency. With that said, the objective of this study is to promote a hermeneutical approach to the Brazilian Government proposals of including medical students in the fight against COVID-19 (Coronavirus Disease 2019) pandemic through an ethical perspective.

Development: The governmental resolutions, published in the Brazilian Official Gazette, were discussed in the light of the Universal Declaration on Bioethics and Human Rights (UDBHR) and the Brazilian Medical Student Code of Ethics (CEEM), as the first one guides the debate through a pluralist, multi-, inter-and transdisciplinary bioethics, and the latter brings specified guidance to the studied population group. To better articulate the discussion, the main measures were subdivided into 3 sections: about the risk assessment; about the participation of $5^{\text {th }}$ - and $6^{\text {th }}$-year students; about the early graduation. In the first one, the creation of participation alternatives has been proposed, including remote participation, without direct contact with patients, aiming to ensure the students' integrity and to maximize the potential positive effects with minimum harm. After that, the predicted obligatory enrollment for undergraduate students attending the final years of medical school and the possibility of obtaining credit hours for the curricular internship in exchange for participation in the strategic action "O Brasil Conta Comigo" were assessed. Finally, the graduation anticipation and the need for a guarantee that the new graduates have the required knowledge and expertise for the medical profession were questioned.
\end{abstract}

Conclusions: For an effective response against the disease, it's necessary to collectively structure the adopted measures, benefiting from the capabilities that the students already have, while respecting their limitations, vulnerabilities, and freedoms. It should also be emphasized that any ethical decisions in the context of Medicine and of future generations of professionals can have immeasurable consequences for these individuals, their patients, and communities and thus, one must ensure that the benefits will be the best and greatest possible.

Keywords: Medical Education; Medical Students; Bioethics; Coronavirus Infections; Pandemics.

${ }^{1}$ Universidade Federal de Pernambuco, Caruaru, Pernambuco, Brasil.

2 Universidade Federal de Pernambuco, Recife, Pernambuco, Brasil.

Editora-chefe: Daniela Chiesa

Editor associado: Roberto Zonato Esteves

Recebido em 20/07/20; Aceito em 23/12/20.

Avaliado pelo processo de double blind review. 


\section{INTRODUÇÃO}

O desenrolar de pandemias ao longo da história da humanidade sempre foi um importante fator para o desenho da evolução social, da mesma forma que guerras, revoluções ou crises econômicas'. Nesses períodos de convulsão social, os padrões éticos podem ser alterados pela necessidade de equilíbrio entre justiça e benefício ${ }^{2,3}$. Diferentemente do que está acontecendo nesta pandemia, na qual a imprensa presta importante colaboração aos gestores de saúde ao informar a população, durante a pandemia da gripe espanhola de 1918 muitas autoridades de saúde se recusaram a divulgar o real quadro de disseminação da doença para evitar pânico e revolta ${ }^{3,4}$.

Situações como essas geram fragilidades nos sistemas de saúde, expressas por incertezas e desconhecimento em torno do principal adversário patogênico e em como combatê-lo, além da inerente urgência por respostas ${ }^{5}$. Isso também se observa no cenário atual, com todas as interrogações que ainda circundam o combate à pandemia da coronavirus disease 2019 (Covid-19). Provocada pelo segundo coronavírus da síndrome respiratória aguda grave (severe acute respiratory syndrome coronavirus 2 SARS-CoV-2), a doença já contaminou mais de 66,6 milhões de pessoas e levou à morte mais de 1,5 milhão ${ }^{6}$, e muitas perguntas ainda continuam em aberto. As evidências são insuficientes, e os relatos das experiências em outros países podem ser bastante divergentes, principalmente no que se refere à educação médica e à forma de esta ser inserida, ou não, nesse contexto.

Desse modo, cada nação conduz a comunidade de estudantes de Medicina da maneira que considera mais coerente. No Brasil, o Ministério da Saúde (MS) publicou o Edital no 4, de 31 de março de 2020, que convoca estudantes dos cursos de graduação em Medicina, Enfermagem, Farmácia e Fisioterapia para o enfrentamento da pandemia, justificando essa decisão como de caráter emergencial7.

Assim, o objetivo deste estudo é analisar, a partir de um exercício hermenêutico e à luz da Declaração Universal sobre Bioética e Direitos Humanos (DUBDH) e do Código de Ética do Estudante de Medicina (CEEM), as propostas do governo federal do Brasil para a inserção de estudantes de Medicina no combate à Covid-19. O fortalecimento desse debate se torna especialmente relevante nessa situação, já que as incertezas do cenário atual podem levar, por pressão da urgência do quadro, à aprovação de condutas que relativizem ou negligenciem princípios éticos 5 .

\section{BREVE HISTÓRICO DA ÉTICA APLICADA À SAÚDE}

Inicialmente, entende-se ser relevante definir o papel da ética e como ela se desenhou com o passar do tempo, em especial na América Latina e no Brasil. Isso é importante porque cada sociedade possui seus próprios valores, a moral, que refletem o que é socialmente aceito ou não ${ }^{5}$. A palavra moral surge do termo latino mos, do qual advém a expressão moralis, criada por Cícero como tradução da palavra grega éthika ${ }^{8}$.

Logo, do ponto de vista etimológico, moral e ética podem ser entendidas como sinônimas, porém sua contextualização histórica resultou em compreensões distintas ${ }^{8}$. Ferrer e Álvarez ${ }^{8}$ fazem a distinção entre vida moral e saber moral, sendo a primeira involuntária, como parte da biografia de cada pessoa, vivenciada a partir dos usos, dos costumes e das tradições da sua sociedade. Já o saber moral somente pode ser adquirido por meio da reflexão crítica sobre a vida moral, que os autores denominam de "ética".

Portanto, a ética é a moral refletida, a ciência que resolve dilemas que possivelmente venham a surgir, analisando intenções, implicações e prováveis consequências ${ }^{5,8}$. No âmbito da medicina, o estudo da ética toma uma importância particular porque o trabalho médico não é meramente técnico, ele atinge profundamente as relações interpessoais e o rumo da saúde pública, campos inesgotáveis de embates morais ${ }^{9,10}$.

Ao longo da história, várias foram as tentativas de adotar códigos ou juramentos como um imperativo ético normativo a ser seguido no exercício médico ${ }^{11}$, a exemplo do Juramento de Hipócrates, que aborda a relação médico-paciente com ênfase no cuidado $^{3}$, de modo impositivo e paternalista ${ }^{12}$. Essa visão vinha acompanhada da ética de virtudes, que persistiu até o surgimento da medicina moderna no fim do século XVIII, quando se sentiu necessidade de guiar as condutas dos profissionais por meio de deveres, e não apenas de faculdades individuais ${ }^{9,10}$. Isso ocorreu porque, como Foucault ${ }^{13}$ descreve, era necessário ligar a medicina ao Estado, punindo abusos profissionais e desvios de moralidade.

Motivado por essa demanda, o inglês Thomas Percival tornou-se o primeiro a publicar um código de ética médica na Modernidade, no ano de 1803 , dando mais um passo a caminho da normatividade ${ }^{10}$. O trabalho de Percival se tornou ainda referência para os primeiros códigos nacionais de ética médica, adaptados para a realidade de cada país ${ }^{10}$.

Contudo, na segunda metade do século XX, após diversos avanços técnico-científicos ${ }^{12}$, já não bastava que a medicina se norteasse apenas por aspectos deontológicos. Era preciso que se voltasse também para o meio ambiente e a sobrevivência humana. Com isso, o médico estadunidense Van Rensselaer Potter, em seu livro Bioethics: bridge to the future, de 1971, sugere a criação de uma disciplina que alie ética e conhecimento biológico, a qual denominou bioética ${ }^{14}$.

Esse novo campo do saber ganha mais força quando relatos de conflitos éticos envolvendo pesquisas com seres humanos são trazidos à tona nos Estados Unidos, obrigando o governo do país a criar uma comissão específica para fiscalizar 
esses estudos. Desse órgão, resultou o Relatório Belmont, que trazia três princípios considerados básicos para qualquer pesquisa biomédica: o respeito à autonomia, a beneficência e a justiça ${ }^{12}$. A estes, foi agregado o princípio da não maleficência com a publicação do livro Principles of biomedical ethics, por Beauchamp e Childress, em 1979, introduzindo a bioética principialista ou principialismo ${ }^{12}$. Contudo, a própria utilização do termo "princípios" gera ambiguidade, já que não fornecem teorias morais ou guias de ação, funcionando mais como ideais éticos ${ }^{12,15}$.

Além disso, sua intenção de gerar ferramentas universais invisibiliza diferenças morais e culturais entre as sociedades no contexto do Sistema Mundo e reforça relações de dominação dos países "desenvolvidos" sobre aqueles "subdesenvolvidos", legitimando uma falsa superioridade intelectual do eixo europeu-estadunidense ${ }^{12}$. Essa pretensa hegemonia da teoria principialista impõe limitações ao debate bioético ao reduzir o conceito original de Potter, de uma bioética multidisciplinar e ambiental, apenas ao seu contexto clínico e científico, tornando-a insuficiente para as discussões de saúde coletiva ${ }^{12,16}$.

Para além do individualismo, os dilemas de saúde coletiva tratam de temas que ultrapassam as relações interpessoais e atingem grandes grupos populacionais nas ações de prevenção, promoção de saúde e qualidade de vida. Além disso, na situação atual, a capacidade de previsão dos efeitos das políticas adotadas pode ser comprometida, tornando insustentável o principialismo, já que não há certeza quanto à possível beneficência ou nocividade das propostas ${ }^{16}$.

Assim, para este estudo, o documento-base que norteará as discussões éticas é a DUBDH, elaborada pela Organização das Nações Unidas para a Educação, a Ciência e a Cultura (United Nations Educational, Scientific and Cultural Organization - Unesco) em 2005, que estende o debate também para os âmbitos ambientais, sanitários e sociais ${ }^{12}$. Essa declaração surge em um contexto de fortalecimento da defesa de uma bioética plural, multi, inter e transdisciplinar, considerando as liberdades fundamentais, a dignidade humana e os tratados internacionais em direitos humanos ${ }^{12,17}$.

Em adição, será também considerado o CEEM de 2018, do Conselho Federal de Medicina (CFM), que traz orientações especiais para o grupo populacional em questão, mesmo que ainda orientadas por um padrão principialista ${ }^{18}$.

\section{A PARTICIPAÇÃO DOS GRADUANDOS DE MEDI- CINA NAS MEDIDAS DE COMBATE À COVID-19}

Em decorrência da necessidade de enfrentamento da Covid-19 e após a declaração nacional de Emergência em Saúde Pública ${ }^{19}$, o MS passou a adotar medidas para contenção do avanço da doença, em cooperação com outros ministérios e instâncias governamentais, em níveis nacional, estadual e municipal.
Dentre essas medidas, foi publicada a Portaria no 492, de 23 de março de 2020, que instituiu a ação estratégica "O Brasil Conta Comigo" com a inclusão dos estudantes da área de saúde ${ }^{20}$. Essa portaria traz um pacote de medidas temporárias que começou a ser executado após o lançamento do Edital no 4, de 31 de março de 2020, convocando graduandos dos cursos de Medicina, Fisioterapia, Enfermagem e Farmácia7.

Além disso, a Presidência da República, por meio da Medida Provisória n 934, de $1^{\circ}$ de abril de 2020, autorizou a antecipação da colação de grau dos estudantes dos cursos já citados, tomando como justificativa a atuação no combate à pandemia ${ }^{21,22}$.

Neste estudo, as principais medidas adotadas na educação médica, publicadas no Diário Oficial da União, serão debatidas com o auxílio das orientações da DUBDH e do CEEM. Analisadas sob o espectro dos estudantes de Medicina, as considerações foram subdivididas em três seções: "Sobre a avaliação de risco"; "Sobre a participação dos alunos do quinto e sexto anos"; "Sobre a antecipação de colação de grau".

\section{Sobre a avaliação de risco}

Antes de quaisquer decisões no campo da saúde pública, faz-se necessária uma avaliação dos riscos inerentes às medidas propostas, também com a intenção de maximizar seus efeitos benéficos e prognosticar e minimizar os efeitos nocivos aos quais os indivíduos estariam sujeitos, conforme defendido nos artigos $4^{\circ}$ e 20 da DUBDH ${ }^{23}$. Seria então a solução mais adequada a inserção do graduando de Medicina na linha de frente do combate a uma epidemia da qual existem poucas evidências?

Historicamente, a inserção desses estudantes para o confronto de crises epidemiológicas traz divergências. Na segunda fase da epidemia de gripe espanhola em 1918, graduandos voluntários foram chamados para suprir as necessidades do sistema de saúde espanhol, em consequência de redução quantitativa significativa dos profissionais de saúde por óbito ${ }^{24,25}$. Já durante o surto de síndrome respiratória aguda grave de 2003 em Hong Kong, a participação de estudantes de Medicina em contato direto com pacientes foi proibida após 17 alunos testarem positivo para a doença, subsequente ao atendimento de um paciente que não tinha indícios de contaminação ${ }^{25,26}$.

Esse risco de contaminação aumentado é inerente à linha de frente e pode ser confirmado com dados epidemiológicos do atual enfrentamento da Covid-19. Até 6 de dezembro de 2020 , cerca de $12,3 \%$ dos casos notificados em Pernambuco ${ }^{27}$ eram de profissionais de saúde. Ou seja, quando se inserem estudantes no contato direto com os pacientes, impõe-se também um risco maior para infecção.

Além disso, os benefícios também são restritos. Por um lado, a inserção dos estudantes poderia elevar a capacidade 
de atendimento do sistema de saúde, conforme idealizado pelo próprio edital, e fornecer aos alunos uma oportunidade única de aprendizado clínico. Por outro lado, sabe-se que a realidade hospitalar atual não segue mais a rotina da graduação como antigamente, enfrentando o esgotamento dos profissionais de saúde $^{28}$ e a suspensão de consultas ambulatoriais, o que torna impróprio o processo de ensino-aprendizagem ${ }^{29}$ planejado nos setores de clínica médica, pediatria e saúde coletiva ${ }^{7}$. Em adição, segundo levantamento de abril de 2020 da Associação Paulista de Medicina (APM), pelo menos metade dos médicos sofrem com falta de equipamentos de proteção individual (EPIs), como máscaras N95, em seus locais de trabalho, pondo em risco a própria segurança para o enfrentamento da pandemia ${ }^{30}$. Dito isso, o racionamento desses equipamentos para permitir o ensino médico eleva o risco de infecção tanto para graduandos e funcionários do serviço quanto para seus familiares. Valeria então se submeter ao risco em prol dos supostos benefícios para a educação?

É possível também que haja outras alternativas para a adesão dos estudantes que considerem suas limitações como graduandos e que diminuam os riscos de contaminação. Algumas atividades podem ser realizadas de modo remoto, como a produção de material digital para educação da comunidade médica e da população em geral. Pode-se incentivar a criação de plataformas com evidências científicas atualizadas e de redes sociais para divulgação de notícias, combate a fake news e resolução de dúvidas da comunidade local e nacional, a exemplo do que está sendo feito pelos estudantes da Harvard Medical School ${ }^{29}$. Os voluntários poderiam também formular protocolos de segurança com as equipes de gestão e regulação e treinar profissionais para o uso racional de $\mathrm{EPIs}^{29}$, entre as várias possibilidades de atuação.

Portanto, acredita-se que a tomada de decisão para a inclusão de estudantes de Medicina nas estratégias de enfrentamento da Covid-19 possui uma justificativa ética utilitarista, compreendendo-se que o gestor público fez uso do cálculo utilitário com a intenção de possibilitar o maior benefício ao maior número de pessoas possível ${ }^{31}$. Contudo, como visto, não se trata de uma equação simples, porque envolve múltiplos atores e fatores intervenientes no processo. Ela deveria ser precedida por uma análise profunda sobre a relação risco-benefício e, após a deliberação, ser ancorada em uma série de medidas de amenização dos riscos, garantindo a integridade pessoal dos estudantes envolvidos e estimulando o uso prudente do melhor de sua capacidade intelectual, conforme defendido pelo artigo $8^{\circ}$ da DUBDH ${ }^{23}$.

\section{Sobre a participação dos alunos do quinto e sexto anos}

Considerando a inserção dos estudantes de Medicina, é importante fazer uma crítica inicial à proposta de participação dos alunos do quinto e sexto anos do curso. Para eles, a inscrição foi posta como obrigatória, tratando como "dever" o cadastro na ação, por meio do ponto 3.2.1 do edital' ${ }^{7}$. Simultaneamente, no ponto 3.2.4.3, imputa-se caráter de consentimento esclarecido a esse mesmo cadastro, valendo "para todos os efeitos jurídicos"7.

Mesmo que portarias não tenham caráter normativo, qualquer intento em ferir as liberdades individuais e desrespeitar a autonomia é antiético e vai contra os artigos $3^{\circ}$ e $5^{\circ}$ da DUBDH, que tratam de dignidade humana e responsabilidades individuais ${ }^{23}$. Além disso, o poder de concordância atribuído ao cadastro obrigatório desses alunos serve verdadeiramente como um contrato ${ }^{10}$ apenas para isentar o Estado "de qualquer culpa caso algo saísse errado no desenvolvimento de suas práticas" (p. 448) ${ }^{12}$.

Seguidamente, é proposta a inserção dos internos nos serviços de clínica médica, pediatria e saúde coletiva, sendo possível a utilização das horas dedicadas para substituição da carga horária do estágio curricular obrigatório dessas disciplinas ${ }^{7,20,22}$. Mais uma vez, deve ser proposta uma avaliação de benefícios e malefícios que tal medida poderia gerar para alimentar o debate ético. Ao passo que a dispensa de carga horária obrigatória pode ser uma recompensa "justa" para os alunos que se voluntariaram a atuar no enfrentamento da pandemia, ela pode também ser injusta, já que não existem oportunidades para a adesão de estudantes que se inserem em grupos de risco para a Covid-19, desconsiderando a equidade de chances de participação e de tratamento, defendidas no artigo 10 da $D U_{B D H}{ }^{23}$. Logo, deveriam ser avaliadas alternativas que viabilizassem sua inscrição caso desejem, afastando-os da assistência direta a pacientes suspeitos de infecção pelo novo coronavírus, conforme recomendado pelo Conselho Nacional de Saúde ${ }^{32}$.

Por sua vez, equivaler essa experiência ao estágio curricular obrigatório pode prejudicar a qualidade do ensino médico, visto que os serviços de saúde não estão em condições regulares de ensino-aprendizagem ${ }^{29}$ e que seus supervisores possam ser despreparados para a preceptoria, já que não está prevista capacitação para tal. Desse modo, a vivência adquirida pode não condizer com os objetivos propostos para o internato médico nas Diretrizes Curriculares Nacionais (DCN) e no Projeto Pedagógico do Curso (PPC), nem com a sua futura prática médica de rotina. Seria benéfico, então, fazer essa equivalência para incentivo à participação no edital?

Entende-se que essa indagação não pressupõe uma resposta fácil, porque todo processo de ensino-aprendizagem sempre estará exposto a possíveis imprevistos, sendo a pandemia apenas um deles. Todavia, levando em consideração a lógica da aplicação da ética utilitarista, todas as consequências das ações deverão ser avaliadas antes de serem deliberadas ${ }^{33}$, o 
que se considera de extrema relevância para se obter uma boa resposta à pergunta aqui formulada.

\section{Sobre a antecipação de colação de grau}

A mais recente medida referente à educação médica no contexto da Covid-19 foi emitida pela Presidência da República, autorizando a formatura antecipada dos estudantes que completarem pelo menos $75 \%$ da carga horária do internato médico ${ }^{21,22}$. Trazendo a decisão para o âmbito mais biomédico da ética profissional, ao permitirem a certificação dos estudantes, as instituições reguladoras responsáveis confirmam que eles estão capacitados formalmente para a atuação profissional, nesse caso, médica e em benefício dos pacientes ${ }^{12}$. Logo, a principal dúvida que surge é: antecipar a colação de grau ainda traria a garantia de que os formandos têm o conhecimento e a perícia necessários para a profissão médica?

Para ponderar os benefícios e os riscos da medida, é importante lembrar que o internato médico representa pelo menos $35 \%$ de toda a carga horária do curso de graduação em Medicina, segundo as DCN de $2014^{34}$, além de ser a etapa educacional com maior número de horas-aula práticas. Reduzir a duração dessa fase, segundo nota do CFM, seria prejudicar o aprendizado e a vivência dos futuros profissionais, e também não traz a garantia de que estes realmente atuarão na linha de frente, já que não há mecanismos expressos para tal ${ }^{35}$.

Por esse motivo, é importante ressaltar que, mesmo a administração pública tendo se utilizado do cálculo utilitário para justificar essa decisão, conforme compreensão anteriormente externada, deve-se considerar que, ao fazer uso da ética utilitarista, torna-se também necessário garantir que o maior benefício alcançado se prolongue por um maior tempo possível - e este último aspecto não está necessariamente assegurado.

\section{CONSIDERAÇÕES FINAIS}

Buscou-se analisar de forma atenta e minuciosa as mais recentes medidas governamentais (à época da submissão do artigo) para o enfrentamento nacional da pandemia da COVID-19 e que implicam mudanças no modo como os estudantes de Medicina se inserem nesse contexto. Os questionamentos suscitados foram construídos com base em uma ética pluralista e refletem possíveis impactos tanto para a educação médica no Brasil quanto para a nossa sociedade, tornando a discussão ética sobre esses temas de fundamental importância para o progresso em prol do bem-estar de todos e em respeito aos direitos, às dignidades e às liberdades humanas. Para o combate eficaz à doença, é necessária uma estruturação coletiva das ações adotadas, beneficiando-se das capacidades que os estudantes já oferecem, mas ainda dentro de suas limitações como graduandos e de suas vulnerabilidades como seres humanos.

Por esse motivo, mesmo reconhecendo o caráter emergencial das ações governamentais de enfrentamento da Covid-19, deve-se ressaltar que quaisquer decisões éticas, no contexto da medicina e das futuras gerações de profissionais, podem ter repercussões inquantificáveis tanto para esses indivíduos quanto para seus pacientes e suas comunidades ${ }^{16,23}$. quando se delibera sobre questões de saúde, deve-se ter a garantia de que os benefícios serão os melhores e maiores possíveis.

\section{CONTRIBUIÇÃO DOS AUTORES}

Cleide Aparecida de Freitas foi a idealizadora do estudo e participou da análise de dados e da redação do artigo. Gustavo Freitas Alves de Arruda e Giovanna Cecília Freitas Alves de Arruda participaram da revisão bibliográfica, da coleta e análise de dados, e da redação do artigo. Saulo Ferreira Feitosa foi o orientador do estudo e participou da elaboração e da revisão final do artigo.

\section{CONFLITO DE INTERESSES}

Os autores declaram não haver conflito de interesses neste estudo.

\section{FINANCIAMENTO}

Declaramos que não houve financiamento para a realização desta pesquisa.

\section{REFERÊNCIAS}

1. Spinney L. How pandemics shape social evolution. Nature. 2019 Oct 15; 574:324-6.

2. Graf WD, Epstein LG, Pearl PL. Practical bioethics during the exceptional circumstances of a pandemic. Pediatr Neurol. 2020 Apr; 108:3-4.

3. Schrader DE. Medical ethics and pandemic emergencies. Bioethics Update. 2018 Jan;4(1): 80-6.

4. Martini M, Gazzaniga V, Bragazzi NL, Barberis I. The Spanish influenza pandemic: a lesson from history 100 years after 1918. J Prev Med Hyg. 2019;60:E64-E67.

5. Esquivel-Guadarrama JA. Pandemia 2020: algunas consideraciones éticas Rev Mex Anestesiol. abr/jun 2020;43:168-72.

6. Johns Hopkins Coronavirus Resource Center. Covid-19 map. Johns Hopkins Coronavirus Resource Center; 2020 [acesso em 6 dez 2020]. Disponível em: https://coronavirus.jhu.edu/map.html.

7. Brasil. Edital no 4, de 31 de março de 2020. Chama os Estados, o Distrito Federal, os Municípios e estabelecimentos de saúde filantrópicos para aderirem à Ação Estratégica "Brasil Conta Comigo", bem como conclama alunos dos cursos de graduação em Medicina, Enfermagem, Farmácia e Fisioterapia para se cadastrarem visando participação na Ação Estratégica "Brasil Conta Comigo", em caráter excepcional e temporário. Diário Oficial da União; $1^{\circ}$ abr 2020. Edição 63, Seção 3, p. 88.

8. Ferrer JJ, Álvarez JC. Para fundamentar a bioética: teorias e paradigmas teóricos na bioética contemporânea. São Paulo: Edições Loyola; 2005. 504 p.

9. Monte FQ. Ética médica: evolução histórica e conceitos. Rev Bioét 2009;17:407-28. 
10. Miranda AG. A história dos códigos de ética médica. Jornal Medicina. maio/jun 2009; Pensar e Dizer:168-72.

11. Soares FJP, Shimizu HE, Garrafa V. Código de Ética Médica brasileiro: limites deontológicos e bioéticos. Rev Bioét. maio/ago 2017;25:244-54.

12. Garrafa V, Martorell LB, Nascimento WF. Críticas ao principialismo em bioética: perspectivas desde o norte e desde o sul. Saúde Soc. 2016;25(2):443-51.

13. Foucault M. O nascimento da clínica. 7a ed. Rio de Janeiro: Forense Universitária; 2011. 250 p.

14. Potter VR. Bioética: ponte para o futuro. São Paulo: Edições Loyola; 2016. $207 \mathrm{p}$.

15. Roqué-Sánchez MV, Macpherson I. Análisis de la ética de principios, 40 años después. Rev Bioét. jun 2018;26:189-97.

16. Schramm FR, Kottow M. Principios bioéticos en salud pública: limitaciones y propuestas. Cad Saude Publica. jul/ago 2001;17:949-56.

17. Rippel JA, Medeiros CA, Maluf F. Declaração Universal sobre Bioética e Direitos Humanos e Resolução CNS 466/2012: análise comparativa. Rev Bioét. dez 2016;24(3):603-12.

18. Conselho Federal de Medicina. Código de Ética do Estudante de Medicina. Brasília: CFM; 2018.52 p.

19. Brasil. Portaria no 188 , de 3 de fevereiro de 2020. Declara Emergência em Saúde Pública de importância Nacional (ESPIN) em decorrência da Infecção Humana pelo novo Coronavírus (2019-nCoV). Diário Oficial da União; 4 fev 2020. Edição 24-A, Seção 1 - Extra, p. 1.

20. Brasil. Portaria n० 492, de 23 de março de 2020. Institui a Ação Estratégica "O Brasil Conta Comigo", voltada aos alunos dos cursos da área de saúde, para o enfrentamento à pandemia do coronavírus (COVID-19). Diário Oficial da União; 23 mar 2020. Edição 56-C, Seção 1 - Extra, p. 4.

21. Brasil. Medida Provisória n० 934, de $1^{\circ}$ de abril de 2020. Estabelece normas excepcionais sobre o ano letivo da educação básica e do ensino superior decorrentes das medidas para enfrentamento da situação de emergência de saúde pública de que trata a Lei no 13.979, de 6 de fevereiro de 2020 . Diário Oficial da União; $1^{\circ}$ abr 2020. Edição 63-A, Seção 1 - Extra, p. 1.

22. Brasil. Portaria no 374 , de 3 de abril de 2020. Dispõe sobre a antecipação da colação de grau para os alunos dos cursos de Medicina, Enfermagem, Farmácia e Fisioterapia, exclusivamente para atuação nas ações de combate à pandemia do novo coronavírus - Covid-19. Diário Oficial da União; 6 abr 2020. Edição 66, Seção 1, p. 66.

23. Organização das Nações Unidas para a Educação, Ciência e Cultura. Declaração Universal sobre Bioética e Direitos Humanos. Lisboa: Unesco; 2006 [acesso em 8 maio 2020]. Disponível em: https://unesdoc.unesco. org/ark:/48223/pf0000146180_por.
24. Trilla A, Trilla G, Daer C. The 1918 "Spanish Flu" in Spain. Clin Infect Dis. 2008 Sept 10; 47:668-673.

25. Farber ON. Medical students can help combat Covid-19: don't send them home. STAT. 2020 Mar 14 [acesso em 12 maio 2020]. Disponível em: https://www.statnews.com/2020/03/14/medical-students-can-helpcombat-covid-19/.

26. Patil NG, Yan YCH. SARS and its effect on medical education in Hong Kong. Med Educ. 2003;37:1127-8.

27. Centro de Informações Estratégicas de Vigilância em Saúde de Pernambuco. Novo coronavírus (Covid-19). Recife: Cievs/PE; 2020 [acesso em 6 dez 2020]. Disponível em: https://www.cievspe.com/novocoronavirus-2019-ncov.

28. Bauchner H, Sharfstein J. A bold response to the Covid-19 pandemic: Medical Students, National Service, and Public Health. JAMA. 2020;323:1790-1.

29. Soled D, Goel S, Barry D, Erfani P, Joseph N, Kochis ME, et al. Medical student mobilization during a crisis: lessons from a Covid-19 medical student response team. Acad Med. 2020 Sept; 95(9):1384-7.

30. Associação Paulista de Medicina. Os médicos e a pandemia do novo coronavírus (Covid-19). São Paulo: APM; 2020 [acesso em 12 maio 2020]. Disponível em: http://associacaopaulistamedicina.org.br/files/2020/ pesquisa-apm-medicos-covid-19-abr2020.pdf.

31. Mill JS. A liberdade/utilitarismo. São Paulo: Martins Fontes; 2000. 278 p.

32. Brasil. Recomendação n 024 , de 20 de abril de 2020. Recomenda ações relativas à atuação de estudantes de saúde em formação no contexto da Ação Estratégica "O Brasil Conta Comigo". Brasília; 20 abr 2020 [acesso em 12 maio 2020]. Disponível em: http://conselho.saude.gov.br/ recomendacoes-cns/1127-recomendacao-n-024-de-20-de-abril-de-2020.

33. Borges ML, Dall'Agnol D, Dutra DV. Ética. Rio de Janeiro: DP\&A; 2002. 142 p.

34. Brasil. Resolução $n^{\circ} 3$, de 20 de junho de 2014. Institui diretrizes curriculares nacionais do curso de graduação em Medicina e dá outras providências. Diário Oficial da União; 23 jun 2014. Seção 1, p. 8-11.

35. Conselho Federal de Medicina. Nota de esclarecimento aos médicos e à população: antecipação de formatura de estudantes de medicina. Brasília; 15 abr 2020 [acesso em 13 maio 2020]. Disponível em: https://portal.cfm. org.br/images/PDF/2020_nota_antecipacao_formatura.pdf. 